\title{
Application of microsatellite markers for genetic conservation and management of Persian sturgeon (Acipenser persicus, Borodin, 1897) in the Caspian Sea
}

\begin{abstract}
A study was conducted to ascertain the genetic structure and the level of heterozygosity of Acipenser persicus in the Caspian Sea. A total of 167 fish were randomly collected from Turkmenistan, Russia and two regions of Iran. The number of alleles of eleven microsatellite markers ranged from 3 to 21 and the mean observed values of heterozygosity were $0.56 \pm$ $0.20,0.64 \pm 0.14,0.67 \pm 0.16$, and $0.64 \pm 0.11$. The observed heterozygosity was lower than the expected levels. The observed low genetic differentiation indicates that all populations are closely related. Hence, inbreeding is a potential problem, which should be taken into consideration in future breeding programs to avoid a further decline in genetic diversity.
\end{abstract}

Keyword: Acipenser persicus; Caspian Sea; Microsatellite; Persian sturgeon; Genetic conservation 